\title{
Embolization for Hemoptysis-Angiographic Anatomy of Bronchial and Systemic Arteries
}

\author{
Vikash Srinivasaiah Setty Chennur ${ }^{1} \quad$ Kumar Kempegowda Shashi ${ }^{1} \quad$ Stephen Edward Ryan ${ }^{1}$ \\ Adnan Hadziomerovic ${ }^{1} \quad$ Ashish Gupta $^{1}$ \\ ${ }^{1}$ Division of Angio-Interventional Radiology, Department of Medical \\ Imaging, University of Ottawa, The Ottawa Hospital, Ottawa, \\ Ontario, Canada

\begin{abstract}
Address for correspondence Ashish Gupta, MD, Division of Angio-Interventional Radiology, Department of Medical Imaging, University of Ottawa, The Ottawa Hospital, 501 Smyth Road,
\end{abstract} \\ Ottawa, ON K1H 8L6, Canada (e-mail: ashgupta@toh.ca).
}

\begin{abstract}
Massive hemoptysis is a potentially fatal respiratory emergency. The majority of these patients are referred to interventional radiology for bronchial artery embolization (BAE). Immediate clinical success in stopping hemoptysis ranges from 70 to $99 \%$. However, recurrent hemoptysis after BAE is seen in 10 to $55 \%$ patients. One of the main reasons for recurrence is incomplete embolization due to unidentified aberrant

Keywords

- bronchial

- embolization

- hemoptysis bronchial and/or non-bronchial systemic arterial supply. This pictorial essay aims to describe the normal and variant bronchial arterial anatomy and non-bronchial systemic arterial feeders to the lungs on conventional angiography; the knowledge of which is critical for interventional radiologists involved in the care of patients with hemoptysis.
\end{abstract}

\section{Introduction}

Massive hemoptysis is a respiratory emergency with a high potential for mortality. Conservative management by medical therapy alone is associated with a mortality of up to $50 \%{ }^{1}$ Although this is improved with emergent surgical management, significant morbidity and mortality (18-40\%) still persist. ${ }^{1,2}$ Bronchial artery embolization (BAE) is the current treatment of choice and is effective in stopping hemorrhage in 85 to $100 \%$ of patients ${ }^{1}$ with a major complication rate of $<12 \%{ }^{3}$

Recurrent hemoptysis following BAE is seen in 10 to $55 \%$ of cases. ${ }^{1}$ One reason for recurrence is incomplete embolization due to undetected supply from aberrant bronchial and/or non-bronchial systemic arterial feeders. ${ }^{4}$ Accordingly, a thorough knowledge of both conventional and potential uncommon sources of arterial supply to the lungs is essential for interventional radiologists to reduce the risk of recurrence and to help guide repeat embolization if needed.

The aim of this pictorial essay is to illustrate normal and aberrant bronchial artery anatomy as well as potential non-bronchial supply from systemic arteries, that may be responsible for hemoptysis, on conventional angiography. Angiographic indicators that can help identify the likely source of bleeding will also be briefly discussed.

\section{Angiographic Anatomy of Bronchial Arteries}

Bronchial arteries are considered normal (orthotropic) in origin if they arise from the descending thoracic aorta between the superior endplate of the fifth thoracic (T5) and inferior endplate of the sixth thoracic (T6) vertebral body, which is observed in $70 \%$ of the population. ${ }^{5,6}$ During angiography, the left main stem bronchus serves as a useful landmark to search for origins of orthotopic bronchial arteries ( - Fig. 1). Typically, bronchial arteries arise from the anterior or anteromedial surface of the aorta, which is in contrast to intercostal arteries that arise from the posterolateral surface. Bronchial arteries enter the lungs through the hila and course along the bronchi before giving rise to terminal branches at the level of the respiratory bronchiole. They exhibit significant variation with respect to their number, origin, and branching pattern. ${ }^{1}$ The four most common patterns encountered were described by Caudwell et al in an autopsy study of 150 cadavers $^{7}$ ( - Fig. 2). However, recent studies ${ }^{8,9}$ using computed tomography (CT) angiography suggest that the Type 2 pattern described by Caudwell et al, with a single bronchial artery on each side, is the most common pattern followed by two right and single left bronchial arteries Caudwell Type 4, (-Fig. 3). Most right bronchial arteries share their origins with an intercostal artery and
License terms

ISSN 2457-0214. 
arise as intercostobronchial artery ( - Fig. $4 \mathrm{~A})$. They are also most likely to give rise to the anterior spinal artery, which is identified by its characteristic hairpin loop ( - Fig. 4B). ${ }^{2}$

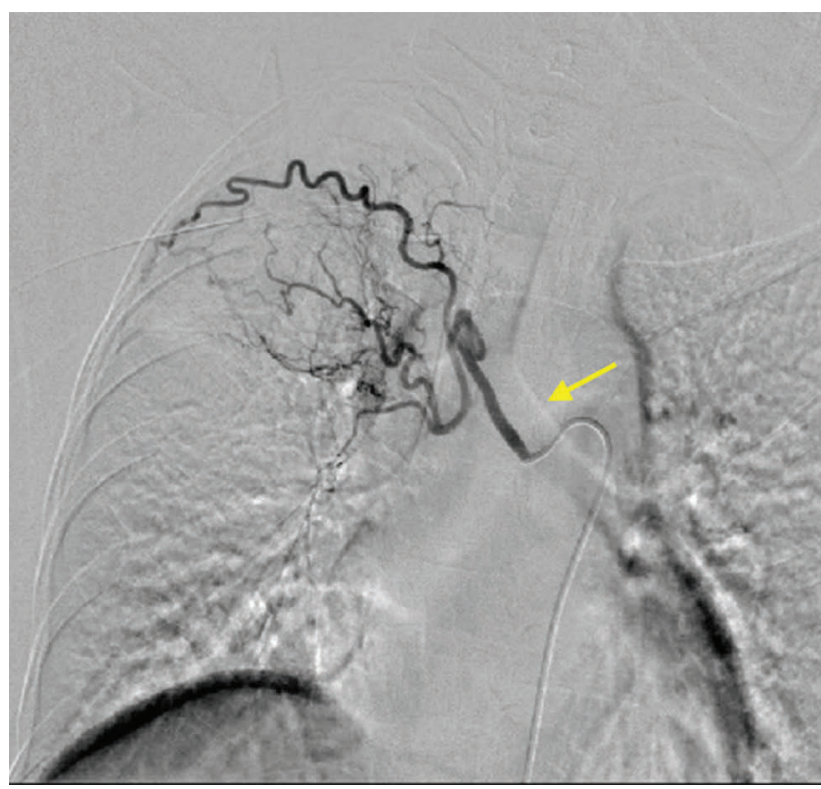

Fig. 1 Right intercostobronchial trunk angiogram for hemoptysis from right lung demonstrates its origin close to left main bronchus (arrow). Latter serves as a useful landmark to search for origins of orthotopic bronchial arteries during angiography.
Bronchial arteries are considered aberrant, anomalous, or ectopic in origin if they arise from the aorta or branches outside the confines of the T5 and T6 vertebral bodies. Like bronchial arteries with orthotopic origin, bronchial arteries with an ectopic origin enter through the hilum and course along the bronchi. Interestingly, an ectopic origin is more common on the right side and in males. ${ }^{9}$ The most common ectopic origin is along the concavity of the aortic arch ( $\boldsymbol{-}$ Fig. $\mathbf{5})$, followed by the subclavian arteries (-Fig. 6) and descending thoracic aorta. Other locations

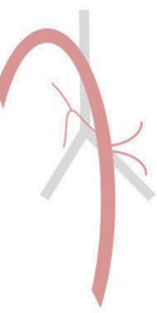

Type 1: $40.6 \%$

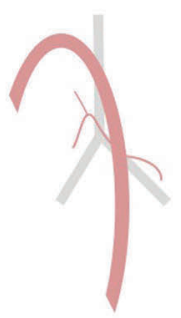

Type 2: 21.3\%

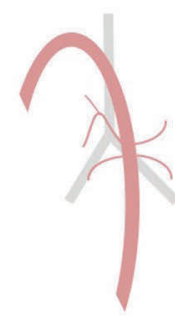

Type 3: $20.6 \%$

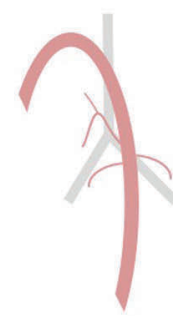

Type 4: $9.7 \%$
Fig. 2 Graphical representation of four most common anatomical patterns of bronchial arteries described by Cauldwell et al. ${ }^{7}$ Type 1 has one right and two left bronchial arteries. Type 2 has one and Type 3 has two bronchial arteries on each side. Type 4 has two right and one left bronchial arteries. Recent studies using $\mathrm{CT}$ angiogram have challenged conclusion of their study. CT, computed tomography.
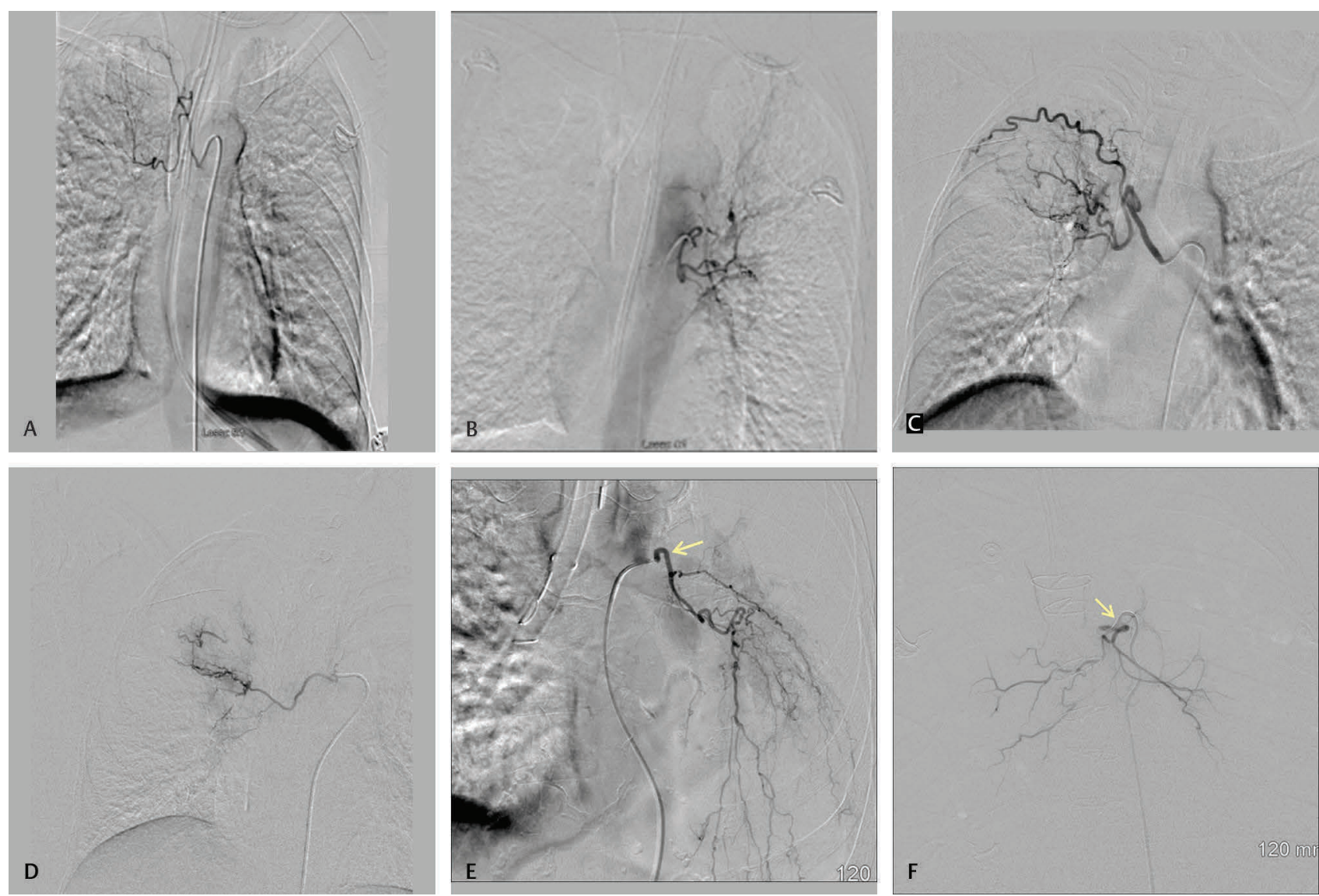

Fig. 3 Common patterns of origin of orthotopic bronchial arteries in different patients showing (A and B) single bronchial artery on each side, (C and D) two right bronchial arteries, (E) single left bronchial artery (arrow), and (F) common bronchial trunk (arrow). 
include the thyrocervical trunk ( - Fig. 7), costocervical trunk, internal mammary and phrenic arteries, abdominal aorta, and the celiac axis or its branches. Rarely, they can arise from the carotid, vertebral, coronary or adrenal arteries. Most ectopic arteries arise in isolation (81\%) rather than as a common bronchial trunk (19\%). ${ }^{8-14}$ Ectopic bronchial arteries can also arise from contralateral aortic branches (-Fig. 6). ${ }^{15}$
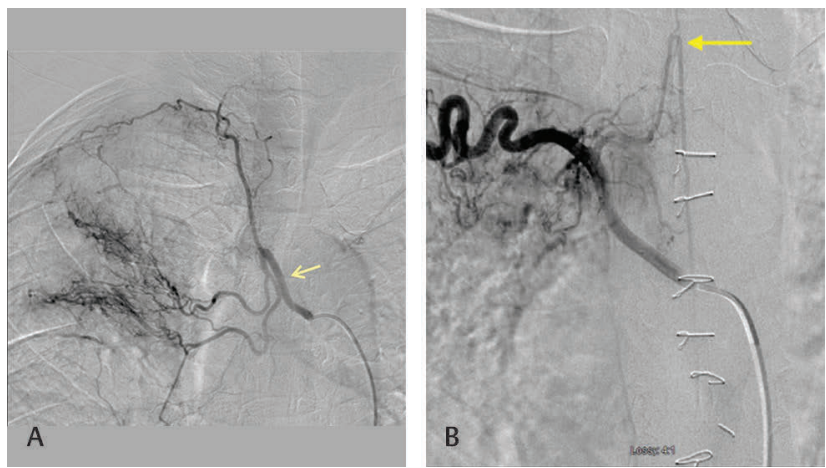

Fig. 4 (A) Majority of right-sided bronchial arteries share their origin with the intercostal artery and arise as an intercostobronchial trunk (arrow). (B) Intercostal arteries can supply spinal cord by their radicular branches or anterior spinal artery. Latter has a characteristic hairpin loop (arrow).

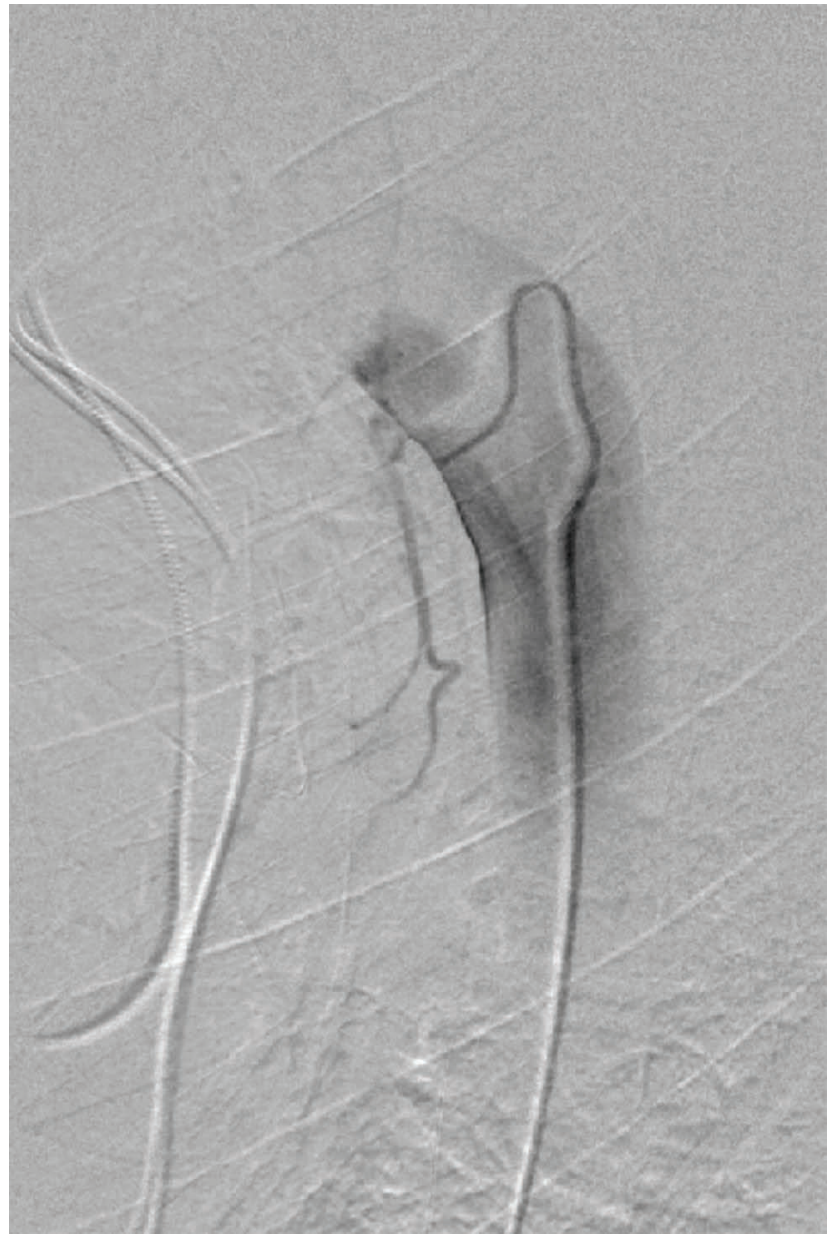

Fig. 5 Angiogram in an 82-year-old male presenting with massive hemoptysis and active bleeding from a lingular segment reveals ectopic origin of the common bronchial artery from distal aortic arch.

\section{Angiography of Non-bronchial Systemic Arteries}

Non-bronchial systemic arterial supply to the lungs is classically seen in patients with chronic inflammatory or infectious lung disease. This is due to recruitment of collaterals from arteries perfusing the structures adjacent to the lung, i.e., pleura, diaphragm, chest wall, etc. Unlike true bronchial arteries (orthotopic or ectopic), these collateral arteries enter through the pleura or pulmonary ligament and do not typically course along the central major bronchi. ${ }^{2,8}$ These collaterals can arise from intercostal arteries (-Fig. 8), branches of the subclavian arteries (internal mammary, thyrocervical, costocervical, thoracodorsal, and lateral thoracic arteries) (Figs. 7C, 9, and 10) or upper abdominal aorta (celiac axis, phrenic arteries, left gastric artery, renal and adrenal arteries, etc.) (-Figs. 11, 12, and 13)

\section{Identifying Source of Bleeding}

Even in patients presenting with ongoing massive hemoptysis, active extravasation is rarely seen on CT or conventional angiography. Angiographic findings that help identify the likely source of bleeding include the presence of tortuous hypertrophied bronchial and non-bronchial systemic arteries, abnormal parenchymal hypervascularity/angiographic blush, bronchopulmonary shunting, and aneurysms (-Fig. 14). Even in the presence of shunting to pulmonary arteries or veins, BAE is considered safe as long as the embolization particles used are larger than $325 \mu \mathrm{m}$ in diameter. ${ }^{2}$

\section{Conclusion}

In summary, BAE is the current treatment of choice for patients with massive hemoptysis. This pictorial essay highlights potential aberrant bronchial and non-bronchial systemic arterial supply to the lungs on conventional angiography. Sound knowledge of such anatomy will reduce initial treatment failure or early recurrence and may help direct repeat embolization if needed.
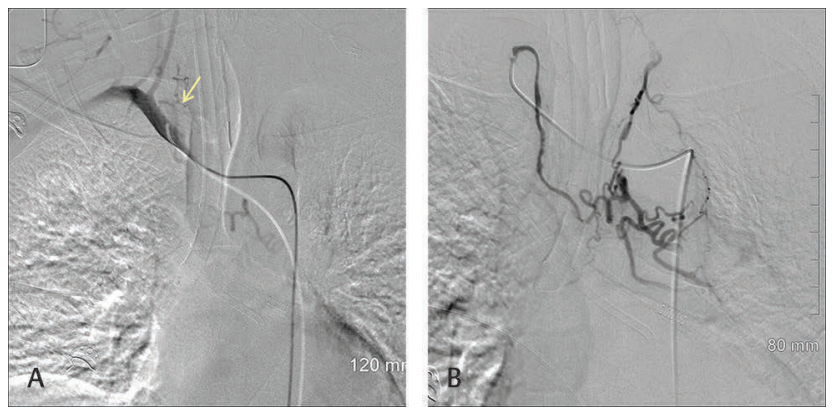

Fig. 6 (A) A 55-year-old male patient with pulmonary tuberculosis presenting with massive hemoptysis and active bleeding from lingula on bronchoscopy following prior embolization. Selective right subclavian angiogram revealed ectopic origin (arrow) of left bronchial artery from the proximal subclavian artery (proximal to vertebral artery which is classically first branch of subclavian arteries. (B) This artery courses in the mediastinum and enters left lung through hilum. 

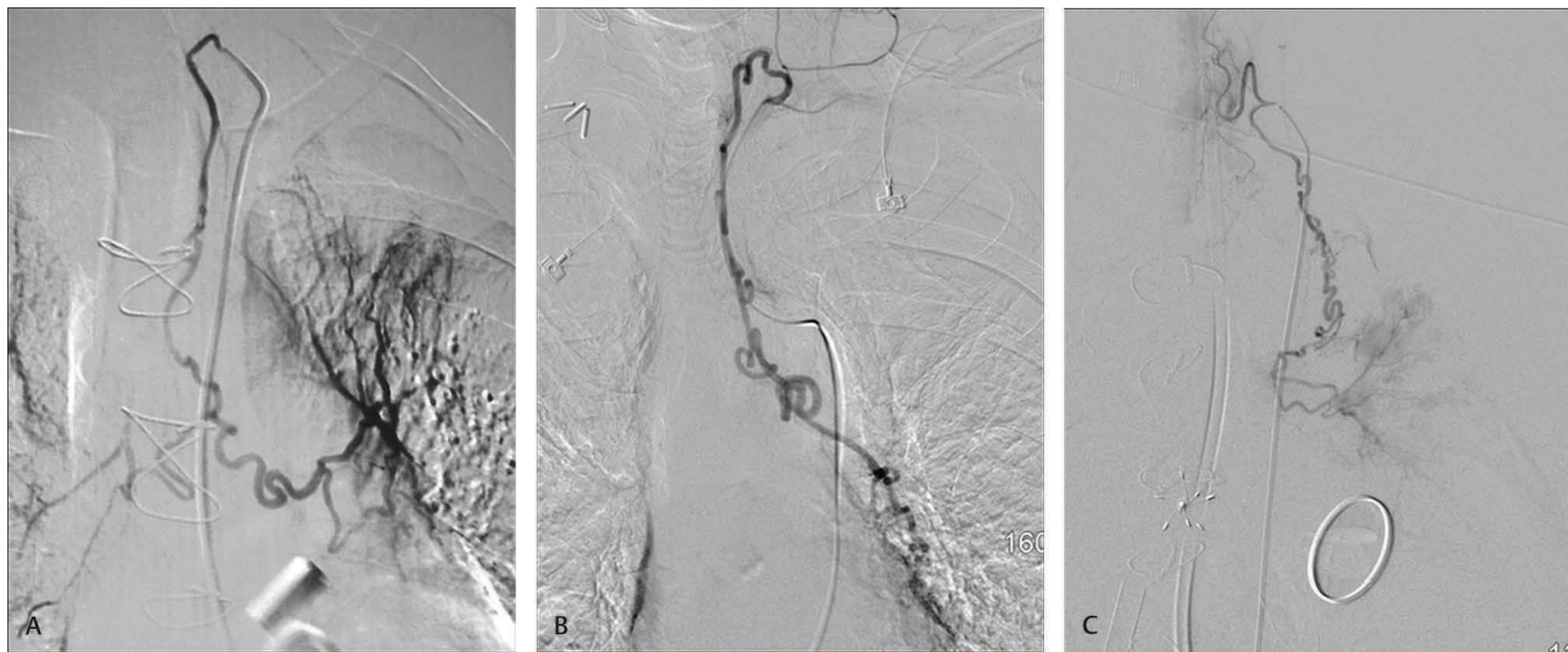

Fig. 7 (A) A common bronchial trunk arising from left thyrocervical artery in a 70-year-old male with bleeding from left lung on bronchoscopy. (B) A 66-year-old male with active bleeding from left lower lobe on bronchoscopy. A left bronchial artery is seen arising from the left thyrocervical artery. (C) Unlike the non-bronchial systemic feeders these arteries course in the mediastinum and then enter the lung through hilum to course along the bronchi.

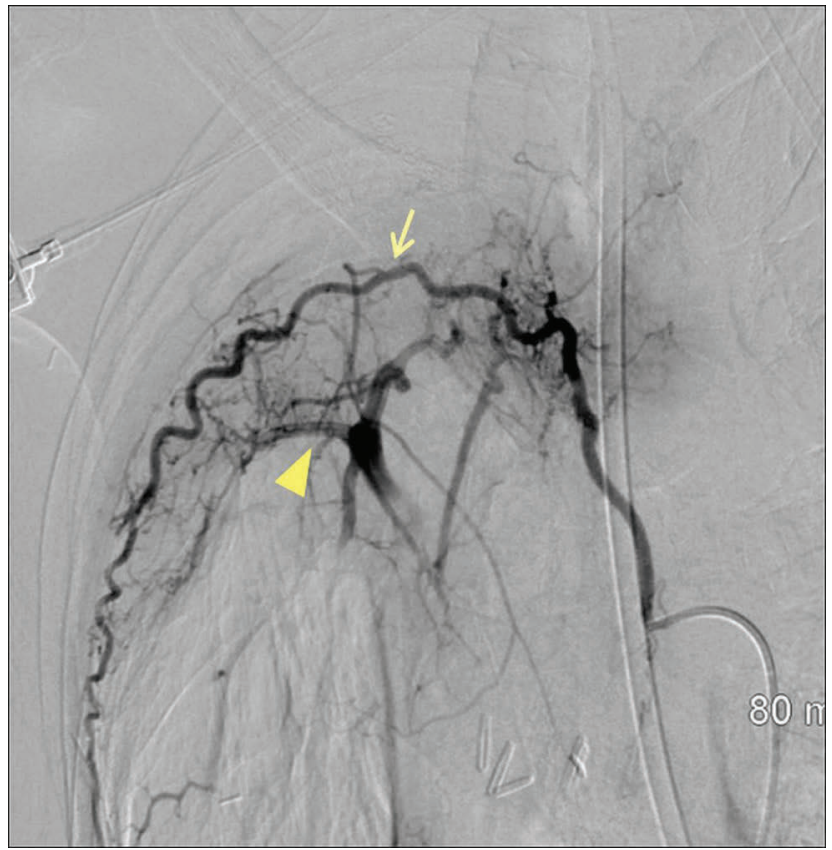

Fig. 8 Bronchial angiogram of a 67-year-old male patient with right upper lobe bronchiectasis showing non-bronchial systemic supply form a hypertrophied, tortuous intercostal artery (arrow) with associated abnormal blush and bronchopulmonary shunting (arrowhead).

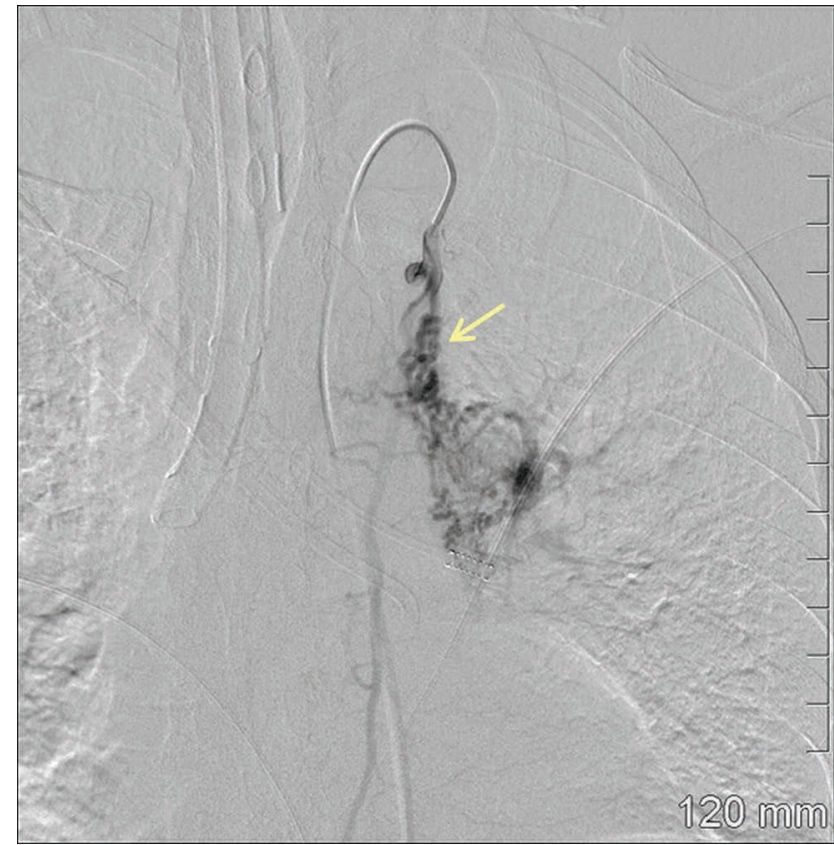

Fig. 9 Collaterals (arrow) arising from left internal mammary artery feeding perihilar region of the left lung in a 56-year-old male presenting with hemoptysis due to bronchiectasis. Unlike true bronchial arteries, these enter through the pleura and do not course along the bronchi. 

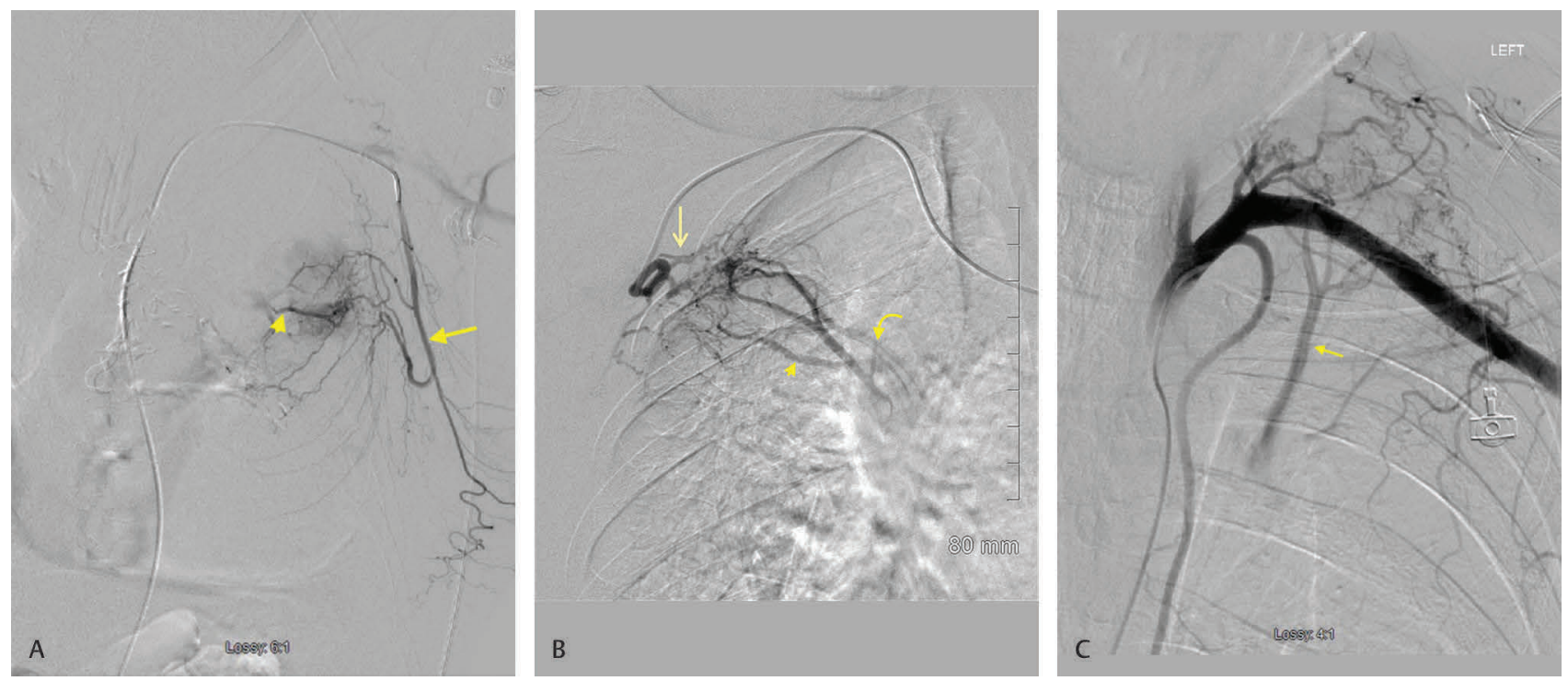

Fig. 10 (A and B) Images of two different patients demonstrating transpleural collaterals from the lateral thoracic arteries (arrows) with associated abnormal blush, and shunting into the pulmonary artery (arrowhead) and pulmonary vein (curved arrow). (C) Left subclavian angiogram in a 63-year-old male presenting with hemoptysis due to left upper lobe pleuroparenchymal fibrosis shows multiple trans-pleural collaterals arising from the left subclavian artery branches with abnormal parenchymal blush and shunting into the pulmonary artery (arrow).
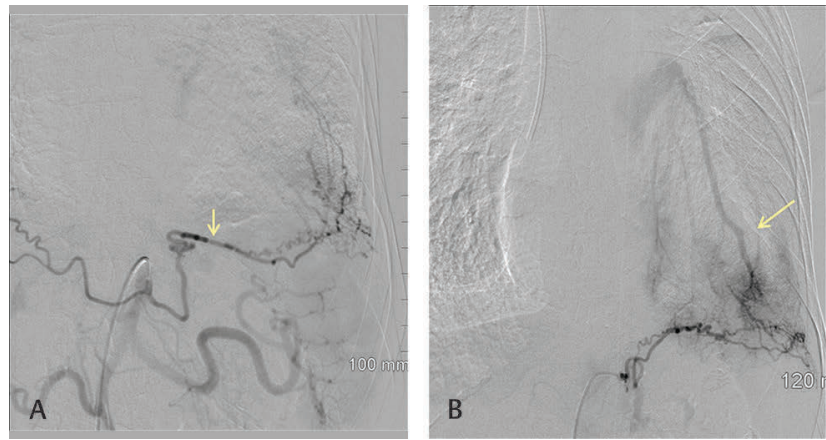

Fig. 11 (A and B) A 72-year-old female patient with hemorrhage within the left lower lobe bronchus shows transpleural collateral supply from left phrenic artery arising from the celiac axis as a common phrenic trunk (arrow in $\mathbf{A}$ ) with bronchopulmonary shunting (arrow in $\mathbf{B}$ ).
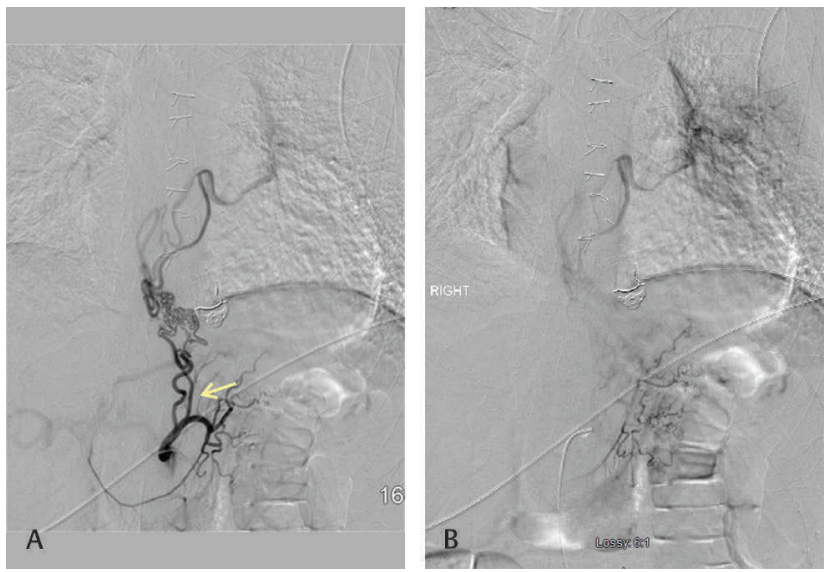

Fig. 12 A 66-year-old male patient with recurrent hemoptysis due to bronchiectasis with collaterals (arrow) arising from the left gastric artery entering the left lung through the pulmonary ligament (A) with an abnormal parenchymal blush (B). 

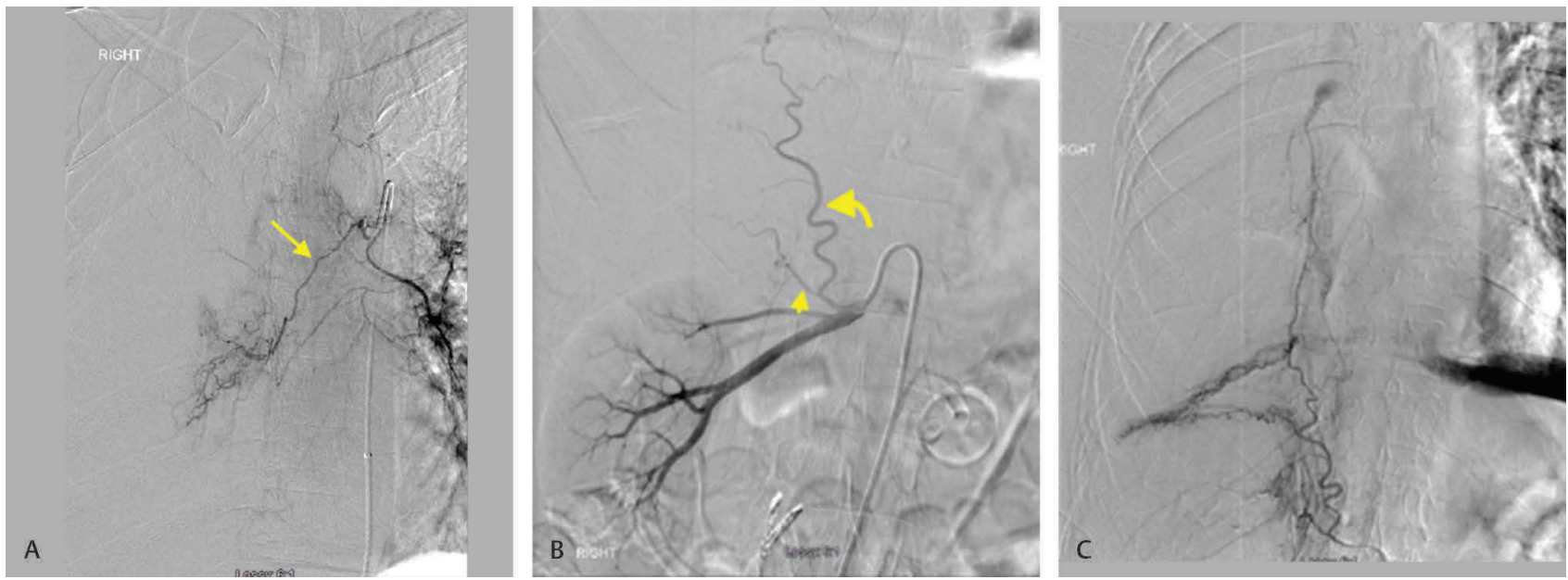

Fig. 13 Massive hemoptysis in a 56-year-old female with complete white out of the right lung and active bleeding from the right lung on bronchoscopy. Patient had embolization of the right bronchial branch (arrow) arising from the orthotopic common bronchial trunk arising from aorta (A). Repeat angiogram due to persistent hemoptysis revealed a collateral (B) arising from the inferior phrenic branch (curved arrow) from right renal artery also supplying the adrenal gland (arrowhead). Transpleural collaterals and pulmonary venous shunting are demonstrated (C).
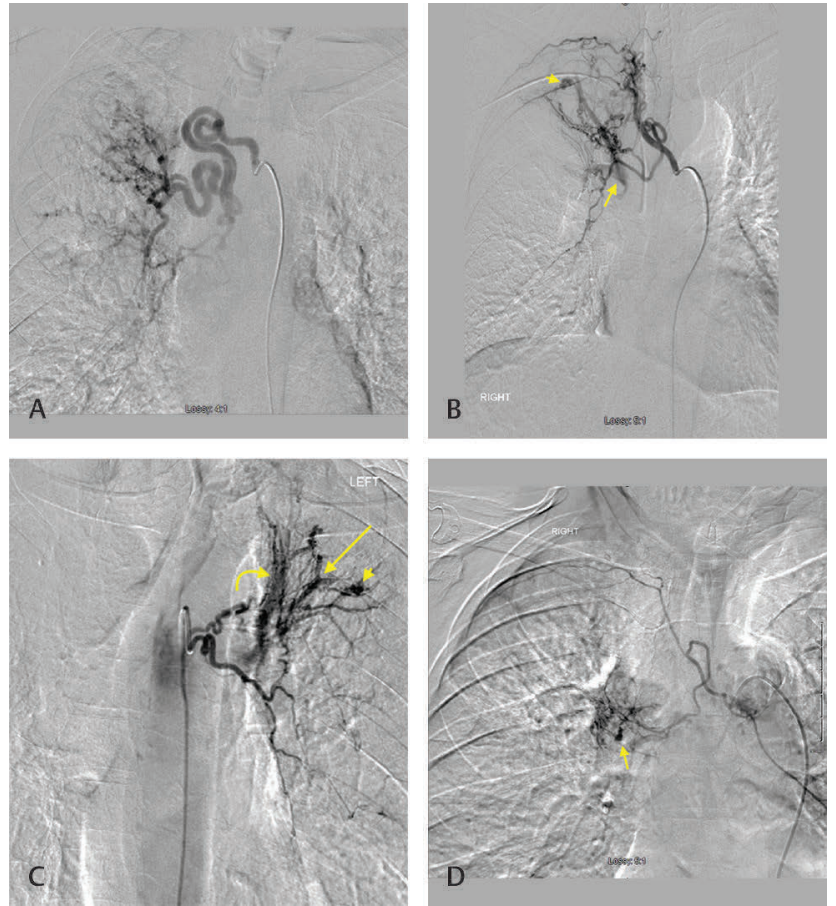

Fig. 14 Markedly hypertrophied right bronchial artery and its upper lobe branch in a 46-year-old male with hemoptysis due to cystic fibrosis (A). Angiogram of the right intercostobronchial trunk in a 55-year-old male with right upper lobe mycetoma reveals hypertrophied bronchial artery as well as an intercostal branch with aneurysmal formation (arrowhead) and shunting to the pulmonary artery (arrow) (B). Left bronchial angiogram in an 81-year-old male patient presenting with left upper lobe fibrocavitary lesion reveals a hypertrophied artery with aneurysmal formation (arrowhead), shunting to pulmonary artery (arrow), and pulmonary vein (curved arrow) (C). Bronchial artery aneurysm (arrow) from the right bronchial artery arising as an intercostobronchial trunk (D).

\section{Conflict of Interest}

All authors have no conflict of interest.

\section{References}

1 Sopko DR, Smith TP. Bronchial artery embolization for hemoptysis. Semin Intervent Radiol 2011;28(1):48-62

2 Yoon W, Kim JK, Kim YH, Chung TW, Kang HK. Bronchial and nonbronchial systemic artery embolization for life-threatening hemoptysis: a comprehensive review. Radiographics 2002;22(6):1395-1409

3 Angle JF, Siddiqi NH, Wallace MJ, et al; Society of Interventional Radiology Standards of Practice Committee. Quality improvement guidelines for percutaneous transcatheter embolization: Society of Interventional Radiology Standards of Practice Committee. J Vasc Interv Radiol 2010;21(10):1479-1486

4 Woo S, Yoon CJ, Chung JW, et al. Bronchial artery embolization to control hemoptysis: comparison of N-butyl-2cyanoacrylate and polyvinyl alcohol particles. Radiology 2013;269(2):594-602

5 Marshall TJ, Jackson JE. Vascular intervention in the thorax: bronchial artery embolization for haemoptysis. Eur Radiol 1997;7(8):1221-1227

6 Stoll JF, Bettmann MA. Bronchial artery embolization to control hemoptysis: a review. Cardiovasc Intervent Radiol 1988;11(5):263-269

7 Cauldwell EW, Siekert RG, Lininger RE, Anson BJ. The bronchial arteries: an anatomic study of 150 human cadavers. Surg Gynecol Obstet 1948;86(4):395-412

8 Battal B, Akgun V, Karaman B, Bozlar U, Tasar M. Normal anatomical features and variations of bronchial arteries: an analysis with 64-detector-row computed tomographic angiography. J Comput Assist Tomogr 2011;35(2):253-259

9 Yener Ö, Türkvatan A, Yüce G, Yener AÜ. The normal anatomy and variations of the bronchial arteries: evaluation with multidetector computed tomography. Can Assoc Radiol J 2015;66(1):44-52 
10 McPherson S, Routh WD, Nath H, Keller FS. Anomalous origin of bronchial arteries: potential pitfall of embolotherapy for hemoptysis. J Vasc Interv Radiol 1990;1(1):86-88

11 Gailloud P, Albayram S, Heck DV, Murphy KJ, Fasel JH. Superior bronchial artery arising from the left common carotid artery: embryology and clinical considerations. J Vasc Interv Radiol 2002;13(8):851-853

12 Jiang S, Sun XW, Jie B, Yu D. Endovascular embolization of an aberrant bronchial artery originating from the vertebral artery in a patient with massive hemoptysis. Cardiovasc Intervent Radiol 2014;37(4):1099-1102
13 Battal B, Saglam M, Ors F, Akgun V, Dakak M. Aberrant right bronchial artery originating from right coronary artery - MDCT angiography findings. Br J Radiol 2010;83(989):e101-e104

14 Hartmann IJ, Remy-Jardin M, Menchini L, Teisseire A, Khalil C, Remy J. Ectopic origin of bronchial arteries: assessment with multidetector helical CT angiography. Eur Radiol 2007;17(8):1943-1953

15 Cohen AM, Antoun BW, Stern RC. Left thyrocervical trunk bronchial artery supplying right lung: source of recurrent hemoptysis in cystic fibrosis. AJR Am J Roentgenol 1992;158(5):1131-1133 